\title{
RESEARCH
}

Open Access

\section{Identification of MMP1 as a potential gene conferring erlotinib resistance in non-small cell lung cancer based on bioinformatics analyses}

\author{
Huyue Zhou ${ }^{1+}$, Qiumei Xiang ${ }^{2+}$, Changpeng Hu' ${ }^{1}$, Jing Zhang ${ }^{1}$, Qian Zhang ${ }^{1 *}$ and Rong Zhang ${ }^{1 *}$ (D)
}

\begin{abstract}
Background: Non-small cell lung cancer (NSCLC) is the major type of lung cancer with high morbidity and poor prognosis. Erlotinib, an inhibitor of epidermal growth factor receptor (EGFR), has been clinically applied for NSCLC treatment. Nevertheless, the erlotinib acquired resistance of NSCLC occurs inevitably in recent years.

Methods: Through analyzing two microarray datasets, erlotinib resistant NSCLC cells microarray (GSE80344) and NSCLC tissue microarray (GSE19188), the differentially expressed genes (DEGs) were screened via R language. DEGs were then functionally annotated by Gene Ontology (GO) analysis and Kyoto Encyclopedia of Genes and Genomes (KEGG) analysis, which up-regulated more than 2-folds in both datasets were further functionally analyzed by Oncomine, GeneMANIA, R2, Coremine, and FunRich.

Results: We found that matrix metalloproteinase 1 (MMP1) may confer the erlotinib therapeutic resistance in NSCL C. MMP1 highly expressed in erlotinib-resistant cells and NSCLC tissues, and it associated with poor overall survival. In addition, MMP1 may be associated with COPS5 and be involve in an increasing transcription factors HOXA9 and PBX1 in erlotinib resistance.
\end{abstract}

Conclusions: Generally, these results demonstrated that MMP1 may play a crucial role in erlotinib resistance in NSCLC, and MMP1 could be a prognostic biomarker for erlotinib treatment.

Keywords: Bioinformatics analyses, NSCLC, Erlotinib resistance, MMP1

\section{Introduction}

Lung cancer is the primary cancer-related death worldwide $[1,2]$. Non-small cell lung cancer (NSCLC) is the main type of lung cancer, including squamous cell carcinoma, adenocarcinoma, and large cell carcinoma, which accounts for approximately $85 \%$ of all cases [3-5]. Currently, chemotherapy plays a crucial role in NSCLC

\footnotetext{
*Correspondence: zhangqianb1102@163.com; xqpharmacylab@126.com ${ }^{\dagger}$ Huyue Zhou and Qiumei Xiang contributed equally to this work. 'Department of Pharmacy, the Second Affiliated Hospital of Army Medical University, 83 Xinqiao Road, Chongqing 400037, China Full list of author information is available at the end of the article
}

treatment for most patients missing the optimal surgical timing or with distant metastases [6]. Platinum-based traditional drug chemotherapy is the standard first-line therapy for patients with advanced lung cancer, which improved the clinical outcomes modestly [7]. However, it causes severe side effects, including nephrotoxicity, neurotoxicity, and emetogenic effect, which usually resulted in a poor quality of life [8]. In recent years, molecular-targeted therapy has been developed into the most promising strategy for NSCLC treatment, which exerts greater anticancer efficacy and fewer side effects than existing chemotherapy [9]. Erlotinib is a specific

(c) The Author(s). 2020 Open Access This article is licensed under a Creative Commons Attribution 4.0 International License, which permits use, sharing, adaptation, distribution and reproduction in any medium or format, as long as you give appropriate credit to the original author(s) and the source, provide a link to the Creative Commons licence, and indicate if changes were made. The images or other third party material in this article are included in the article's Creative Commons licence, unless indicated otherwise in a credit line to the material. If material is not included in the article's Creative Commons licence and your intended use is not permitted by statutory regulation or exceeds the permitted use, you will need to obtain permission directly from the copyright holder. To view a copy of this licence, visit http://creativecommons.org/licenses/by/4.0/. The Creative Commons Public Domain Dedication waiver (http://creativecommons.org/publicdomain/zero/1.0/) applies to the data made available in this article, unless otherwise stated in a credit line to the data. 
inhibitor of epidermal growth factor receptor (EGFR) tyrosine kinase, which is given for locally advanced or metastatic non-small cell lung cancer treatment [10-12]. It can block EGFR downstream signaling pathways such as the signal transducer and activator of transcription 3 (STAT3) and mitogen-activated protein kinase (MAPK) pathway [13]. Although erlotinib has been confirmed to improve the prognosis of NSCLC patients, the majority of these patients inevitably develop acquired resistance with the prolonged treatment time [14, 15]. Several mechanisms of erlotinib resistance have been identified, including T790M gatekeeper EGFR-mutation, activation of bypass-signaling (amplification of IGF1R and MET) [16-20]. Besides, $40-60 \%$ of acquired resistance mechanism is EGFR T790M mutation in first generation EGFR-TKIs [21]. However, the erlotinib acquired resistance mechanisms for NSCLC patients without T790M mutation is not clear.

Genomics microarray, a high-throughput platform, represents a great powerful technology to analyze gene expression comprehensively [22, 23]. In combination with multiple bioinformatics analyses, microarray technology has been widely valued as a tool with great promise to obtain gene signals during tumor process, drug resistance, and verify prognostic biomarkers in cancers [24-26]. Detection and verification of vital targets of drug resistance by using microarray analysis is a novel research strategy and may in favor of uncovering the underlying mechanisms of drug resistance.

Here, we acquired NSCLC of erlotinib resistance and sensitive microarray (GSE80344) and NSCLC adjacent and tumor tissue microarray (GSE19188) from the Gene Expression Omnibus (GEO) online database (https:// www.ncbi.nlm.nih.gov/geo/), and these microarrays were analyzed by bioinformatics methods. To functionally annotate the differentially expressed genes (DEGs), we employed the Gene Ontology (GO) analysis and Kyoto Encyclopedia of Genes and Genomes (KEGG) analysis. Moreover, we further functionally analyzed the genes which up-regulated more than 2-folds in both datasets by Oncomine, GeneMANIA, R2 Coremine, and FunRich to identify that MMP1 was a crucial gene of erlotinib resistance in NSCLC. Meanwhile COPS5 was related to MMP1, and both of their high expressions affected patients' survival. Also, transcription factors HOXA9 and PBX1 may play an important role in this process. Our study provides a potential target and associated mechanisms of erlotinib resistance and suggests that MMP1 could be a prognostic biomarker to erlotinib treatment.

\section{Materials and methods Microarray data}

Two high-throughput sequencing and expression profiling microarray datasets were obtained from the GEO database (https://www.ncbi.nlm.nih.gov/geo). In detail, GSE80344 $(n=16)$ is composed of 12 erlotinib-resistant and 4 erlotinib-sensitive samples in NSCLC and GSE19188 ( $n=156,3$ datasets were abandoned) consists of 91 human NSCLC and 62 adjacent normal tissues. The platforms are GPL16699 and GPL570, respectively.

\section{Processing of microarray data}

The matrix files were downloaded using the $\mathrm{R}$ language, and the adjacent and tumor tissue gene expression profiles (GSE19188) were analyzed using the Lima software package to obtain DEGs. DEGs in the NSCLC erlotinibresistant gene expression profiles (GSE80344) were subsequently analyzed using GEO2R (https: / /www.ncbi. $\mathrm{nlm}$. nih.gov/geo/geo $2 \mathrm{r} /$ ). GEO2R is an online tool to compare two or more groups of samples to identify genes which differentially expressed across experimental conditions. The DEGs cut-off criteria were set to $P$ values $<0.01$ and $\left|\log _{2} \mathrm{FC}\right| \geq 2$ (differential expression multiples $\geq 4$ ), where $\log _{2} \mathrm{FC} \geq 2$ is upregulated, $\log _{2} \mathrm{FC} \leq$ -2 was downregulated, and the error rate was reduced using the Benjamini \& Hochberg method. And a heatmap of the DEGs was produced using Heml 1.0.3.7 heatmap illustrator software and it showed the top 50 upregulated and downregulated genes, respectively. All significant DEGs were shown in a volcano plot produced by $\mathrm{R}$ software.

\section{Functional and pathway enrichment analyses}

Gene ontology analysis is a generally used method for biological functional studies of high-throughput genomics information and transcriptome data [27]. It primarily annotates three ontologies: biological process (BP), cellular component (CC), and molecular function (MF). Kyoto Encyclopedia of Genes and Genomes (KEGG) stores a large amount of data on gene interactions and is commonly used in the signal pathway analysis [28]. To perform functional annotations on the up-expression DEGs, we subsequently conducted the Database, Visualization and Integrated Discovery (DAVID, https:// david.abcc.ncifcrf.gov/), to process the gene ontology and Kyoto Encyclopedia of Genes and Genomes (KEGG) signal pathway analysis. As a result, $P<0.01$ was considered statistically significant.

\section{Expression level analysis}

The erlotinib sensitive and resistant NSCLC dataset GSE38121, GSE69181 and GSE80344 were obtained from the GEO database (https://www.ncbi.nlm.nih.gov/ geo), and analyzed by GEO2R ( $\mid \log _{2}$ (Fold change) $\mid>1$ and $P<0.01$ ). And the expression data (GSE7670 and GSE10072) of MMP1 in normal tissues and tumor tissues were obtained by the online analysis website Oncomine (https://www.oncomine.org/resource/login.html). 
In detail, GSE7670 $(n=57)$ is composed of 30 normal lung tissues and 27 lung adenocarcinoma tissues, and GSE10072 consists of 49 normal lung tissues and 58 lung adenocarcinoma tissues. And the screening conditions were $\mid \log _{2}$ (Fold change) $\mid>2$ and $P<0.0001$. To confirm the expression level of MMP1, GEPIA2 online database was applied based on TCGA and GTEx data. The lung adenocarcinoma (LUAD) and lung squamous cell carcinoma (LUSC) datasets were selected, and genes with $\mid \log _{2}$ (Fold change) $\mid>2$ and $P<0.01$ were considered significant. We also analyzed the protein expression of the hub genes, COPS5, between NSCLC and normal lung tissues using the Human Protein Atlas (HPA, https://www.proteinatlas.org) database.

\section{Survival analysis}

The NSCLC patients were respectively separated into high expression group and low expression group according to MMP1 expression levels, Kaplan-Meier plotter (KM plotter, https://www.kmplot.com) was used to estimate the effect of MMP1 on the survival of patients [29]. The dataset GSE50081 is composed of 127 lung adenocarcinoma patients, and GSE31210 consists of 226 lung adenocarcinoma patients. And the method excluding biased arrays was adopted to control array quality. In the GEPIA2 online database (http://gepia2.cancer-pku. $\mathrm{cn} /$ ), the survival analysis was performed based on 144 lung adenocarcinoma (LUAD) patients' data of a multigene signature (MMP1 and COPS5).

\section{Analyses of MMP1 interactions}

Functional network predictive analysis of MMP1 was performed using the GeneMANIA (https://www.genemania.org/), which is an online gene functional analysis software used to generate hypotheses of gene function, analyze gene lists, and prioritizes genes for functional assays [30]. After entering MMP1 into the search bar and selecting humans from optional organisms, the results show 20 genes closely related to MMP1. At the same time, the R2 tool was used to analyze the correlation of proteins interacting with MMP1. In addition, the biological processes of MMP1, erlotinib, and drug resistance (neoplasm) were further annotated via consulting the Coremine Medical online Database (https://www. coremine.com/medical/).

\section{Identification of transcription factors targeting MMP1}

FunRich (https://www.funrich.org) is a stand-alone software tool used mainly for interaction network analysis of genes and proteins [31]. In this study, we predicted and analyzed the transcription factors targeting MMP-1 by using FunRich.

\section{Statistical analysis}

The GSE80344 data set was processed using the statistical method Benjamini \& Hochberg (false discovery rate, FDR) in GEO2R, and the differential genes were screened using the SAM method. The two groups were compared by the two-tailed Student's T-test. The difference was considered statistically significant at $P<0.05$. GO enrichment analysis and KEGG signaling pathway analysis were performed using statistical tools provided by the DAVID database, adopting the Bonfeerrni, Benjamini false discovery rate, Bootstrap, and Fisher's exact test. All the statistical analyses were conducted with the $\mathrm{R}$ language and SPSS 22.0 software.

\section{Results}

Identification and functional characterization of Upregulated DEGs in Erlotinib resistant NSCLC cells

Erlotinib, an epidermal growth factor receptor (EGFR) tyrosine kinase inhibitor, is an oral targeted anticancer drug for non-small cell lung cancer (NSCLC) treatment, and showing a significant improvement of survival in NSCLC [32]. However, erlotinib-acquired resistance is a tough obstacle to effectively treating NSCLC patients with EGFR mutant characteristics [33-35]. The mechanism underlying erlotinib resistance in NSCLC has not been fully investigated. In this study, we first explored the potential genes conferring erlotinib resistance in the NSCLC erlotinib resistance and sensitive microarray (GSE80344). 308 DEGs in this microarray were identified $\left(P<0.01\right.$ and $\left|\log _{2} \mathrm{FC}\right| \geq 2$ as the threshold cutoff) and a volcano map was shown in Fig. 1a. Among these DEGs, there were 73 significantly upregulated and 235 markedly downregulated (Fig. 1b; Table S1). To characterize the potential role of these significantly upregulated DEGs, these DEGs were first performed for a GO enrichment analysis, results were divided into three ontologies, including biological process (BP), cellular component (CC) and molecular function (MF), and the top five $\mathrm{GO}$ enrichment terms of each ontology were shown in Fig. 1c; Table S2. In the BP category, upregulated DEGs were enriched in branching involved in salivary gland morphogenesis, retinal metabolic process, inflammatory responses, cytokine-mediated signaling pathway, and immune response. In the CC category, the upregulated genes were mainly enriched in the extracellular region, collagen type IV trimer, collagen trimer, and plasma membrane. In the MF category, the DEGs were enriched in chromatin binding, indanol dehydrogenase activity, ketosteroid monooxygenase activity, trans-1,2-dihydrobenzene-1,2-diol dehydrogenase activity, and phenanthrene 9,10-monooxygenase activity. Moreover, KEGG pathway enrichment analyses were further employed to investigate the functional annotations of these upregulated DEGs. Results showed that 


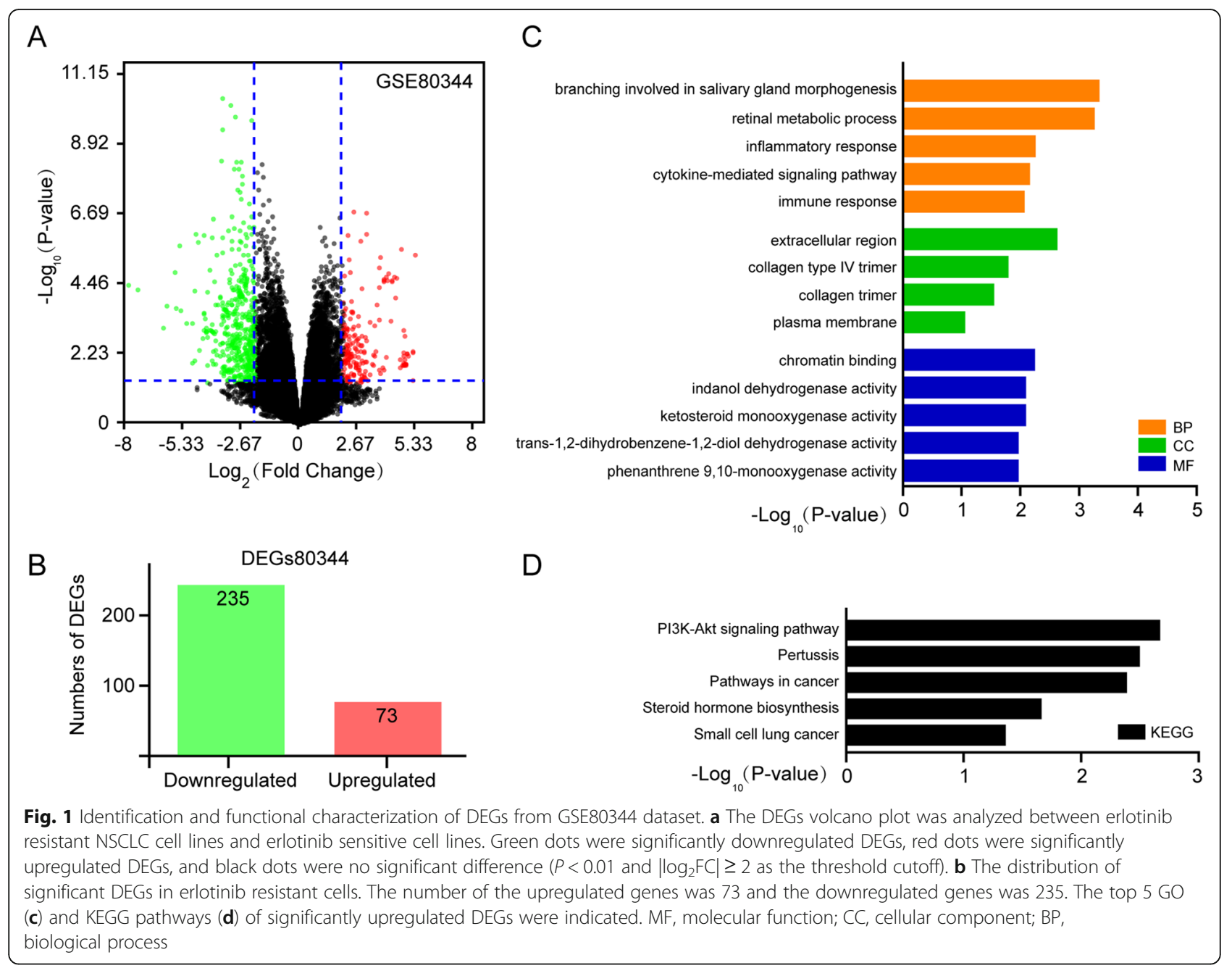

these upregulated DEGs were primarily involved in the PI3K-Akt signaling pathway, pertussis, pathways in cancer, steroid hormone biosynthesis, and small cell lung cancer (Fig. 1d; Table S3).

\section{MMP1 was identified as the gene conferring Erlotinib resistance in NSCLC}

Whether these upregulated DEGs in the erlotinibresistant GSE80344 dataset are also highly expressed in other NSCLC datasets, we further analyzed a dataset GSE19188 ( $n=156,3$ datasets were abandoned) consisting of 91 human NSCLC and 62 adjacent normal tissues. A total of 369 DEGs in dataset GSE19188 were identified by $\mathrm{R}$ language analysis with $P<0.01$ and threshold cutoff $\left|\log _{2} F C\right| \geq 2$, a volcano map was shown in Fig. 2a. Among these DEGs, 122 genes were dramatically upregulated and 247 genes were downregulated (Fig. 2b; Table S4). These upregulated DEGs were further functionally annotated by GO analyses, and the top five GO enrichment terms of BP, CC, and MF categories were shown in Fig. 2c; Table S5. In the BP category, upregulated
DEGs were enriched in microtubule-based movement, mitotic cytokinesis, chromosome segregation, mitotic spindle assembly, and positive regulation of ubiquitinprotein ligase activity. In the CC category, the upregulated genes were mainly enriched in kinesin complex, midbody, spindle pole, condensed chromosome kinetochore, and spindle microtubule. In the MF category, the upregulated DEGs were enriched in microtubule motor activity, ATP binding, ATP-dependent microtubule motor activity, ATPase activity, and ubiquitinconjugating enzyme activity. In addition, KEGG analysis results showed that these upregulated DEGs were mainly involved in signaling pathways of the cell cycle, progesterone-mediated oocyte maturation, ECMreceptor interaction, microRNAs in cancer and rheumatoid arthritis (Fig. 2d; Table S6). Expanded view of the expression of top 50 upregulated and downregulated genes of NSCLC tissues was shown in heatmap (Fig. 2e).

We then combined the upregulated genes of GSE19188 and GSE80344 datasets to narrow down the potential genes conferring erlotinib resistance in NSCLC 
A

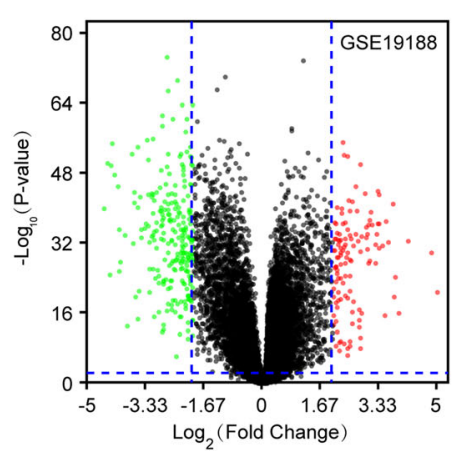

B

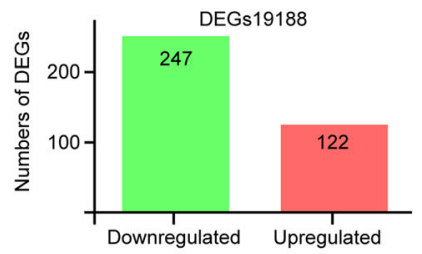

C
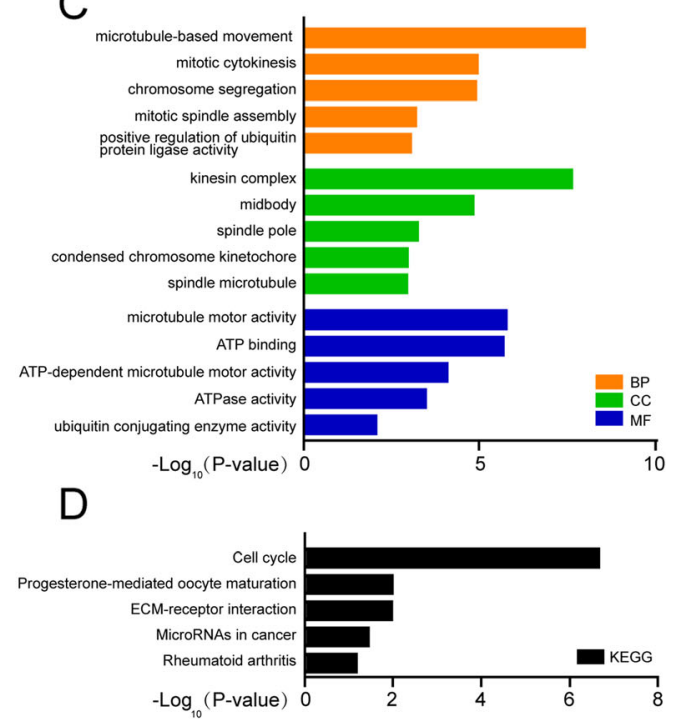

$\mathrm{E}$

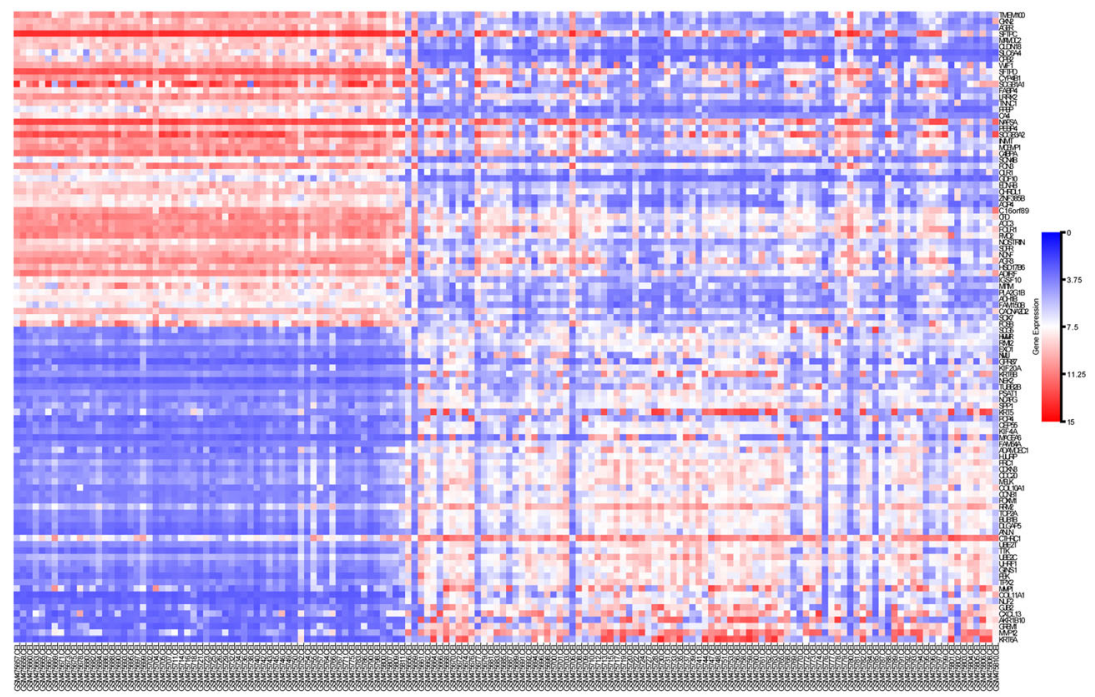

Adjacent normal tissue

NSCLC tissue

Fig. 2 Identification and characterization of DEGs from GSE19188 dataset. a Volcano plot of DEGs between NSCLC tissues and the adjacent normal tissues. Green dots, significantly downregulated DEGs in NSCLC tissues; red dots, significantly upregulated DEGs in NSCLC tissues; black dots, no significant difference $\left(P<0.01\right.$ and $\left|\log _{2} \mathrm{FC}\right| \geq 2$ as the threshold cutoff). $\mathbf{b}$ The distribution of significant DEGs in NSCLC tissues. The number of the upregulated genes was 122 and the downregulated was 247. The top 5 GO (c) and KEGG pathways (d) of significantly upregulated DEGs were indicated. e Heatmap of top 50 upregulated and downregulated DEGs. Red, upregulation; blue, downregulation. MF, molecular function; CC, cellular component; BP, biological process

and found that the expression of MMP1 was markedly increased in both datasets (Fig. 3a). To further validate the expression of MMP1 between erlotinib sensitive and resistant NSCLC cells, 3 datasets were analyzed by GEO2R ( $\log _{2}$ (Fold change) $\mid>1$ and $P<0.01$ ) (Fig. 3b). The results revealed that MMP1 was significantly upregulated in the erlotinib resistance dataset GSE38121, GSE69181 and GSE80344. And we verified that the expression of MMP1 in NSCLC tissues was significantly higher than that in normal tissues, via the datasets GSE7670 $(P=8.01 \mathrm{E}-10$, Fold Change $=21.925)$ and GSE10072 $(P=1.12 \mathrm{E}-15$, Fold Change $=7.277$ ) in Oncomine (Fig. $3 \mathrm{c}$ and d). In addition, to further verify the expression level of MMP1, we utilized another online analysis tool, GEPIA2. And the results revealed that MMP1 was significantly upregulated in lung adenocarcinoma (LUAD) and lung squamous cell 
A

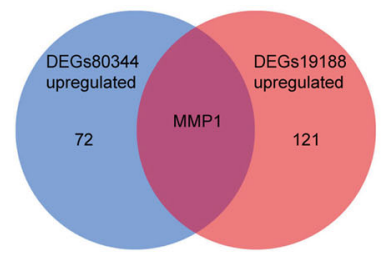

D

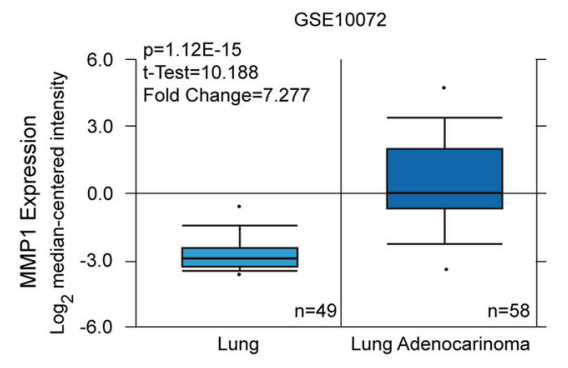

F

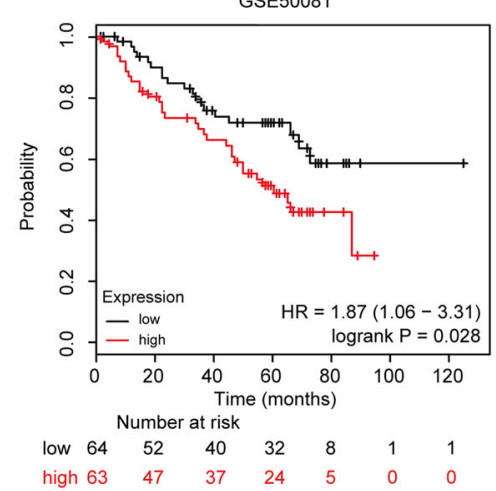

B

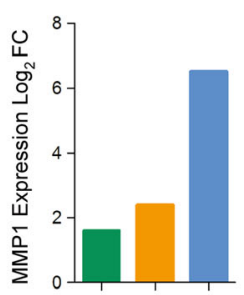

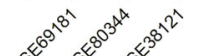

E

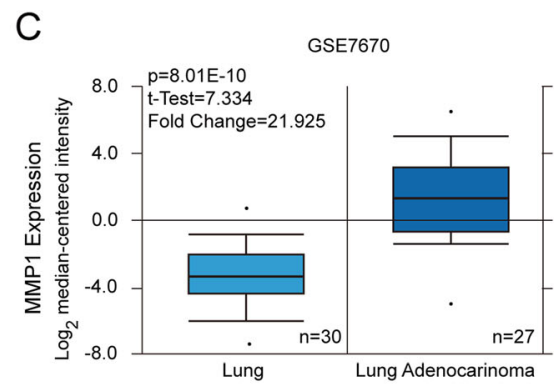

E

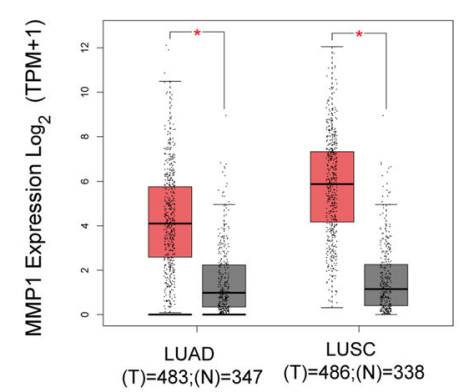

G

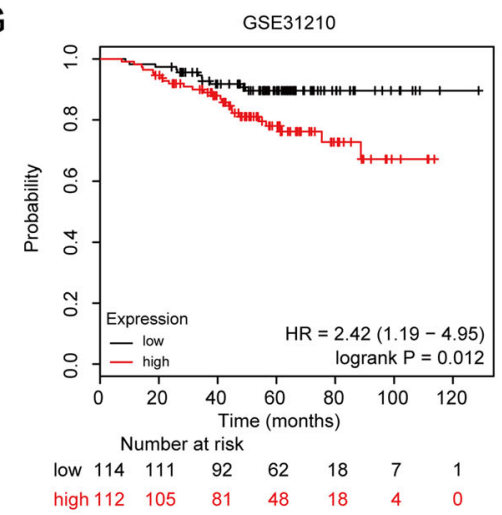

Fig. 3 MMP1 is identified as a potential gene conferring erlotinib resistance in NSCLC. a Venn diagram of significantly upregulated DEGs in GSE80344 and GSE19188. $\mathbf{b}$ The expressions of MMP1were verified through datasets GSE38121 ( $\left.P=2.06 \mathrm{E}-8, \log _{2} \mathrm{FC}=6.52\right), \mathrm{GSE} 69181\left(P=2.25 \mathrm{E}-7, \mathrm{Log}_{2} \mathrm{FC}=1.61\right)$ and GSE80344 ( $\left.P=5.67 \mathrm{E}-3, \log _{2} \mathrm{FC}=2.39\right)$ in erlotinib resistant NSCLC cells. The expressions of MMP1were verified through datasets c GSE7670 $(P=8.01 \mathrm{E}-10$, Fold Change $=21.925)$ and $\mathbf{d}$ GSE10072 $(P=1.12 \mathrm{E}-15$, Fold Change $=7.277)$ in NSCLC tissues and normal lung tissues. e Box-plot revealing that the expression levels of MMP1 is significantly different between NSCLC (LUAD and LUSC) tissues and normal lung tissues by the GEPIA2 online tool ( $\mid \log _{2}$ (Fold change) $\mid>2$ and $P<0.01$ ). f and $\mathbf{g}$ Survival analysis of MMP1 in NSCLC patients was obtained from KM plotter (GSE50081, HR $=1.87$ (1.06-3.31), log-rank $P=0.028 ; G S E 31210, H R=2.42$ (1.19-4.95), log-rank $P=0.012$ ). LUAD, lung adenocarcinoma; LUSC, lung squamous cell carcinoma

carcinoma (LUSC) compared with normal lung tissues ( $\log _{2}$ (Fold change) $\mid>2$ and $P<0.01$ ) (Fig. 3e). In addition, survival analysis by Kaplan-Meier plotter indicated that patients with high expression of MMP1 were associated with poor overall survival (GSE50081, HR = 1.87 (1.06-3.31), log-rank $P=0.028$; GSE31210, HR $=2.42$ (1.19-4.95), log-rank $P=0.012$ ) (Fig. 3f and g).

\section{Validation of MMP1 hub genes related to erlotinib resistance in NSCLC}

To determine how MMP1 plays functions in the erlotinib resistance of NSCLC, the GeneMANIA analysis revealed that MMP1 is closely related to 20 proteins/ genes (Fig. 4a). Among these genes, four genes play crucial regulatory roles in drug resistance in a variety of cancer types. Among these four genes, Integrin subunit alpha 2 (ITGA2), Basigin (BSG), and Thyroid hormone receptor interactor 6 (TRIP6) were downregulated in the erlotinib resistance dataset GSE80344, while COP9 signalosome subunit 5 (COPS5) was upregulated in this dataset (Fig. 4b). Considering that gene expression was not always consistent with its protein level, we further analyzed the protein level of COPS5 in NSCLC tissues from the Human Protein Atlas (HPA) database. The 

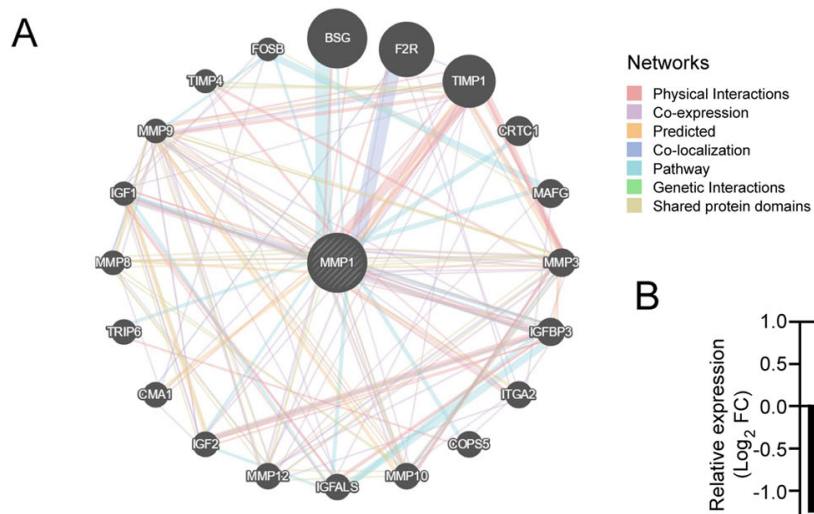

B
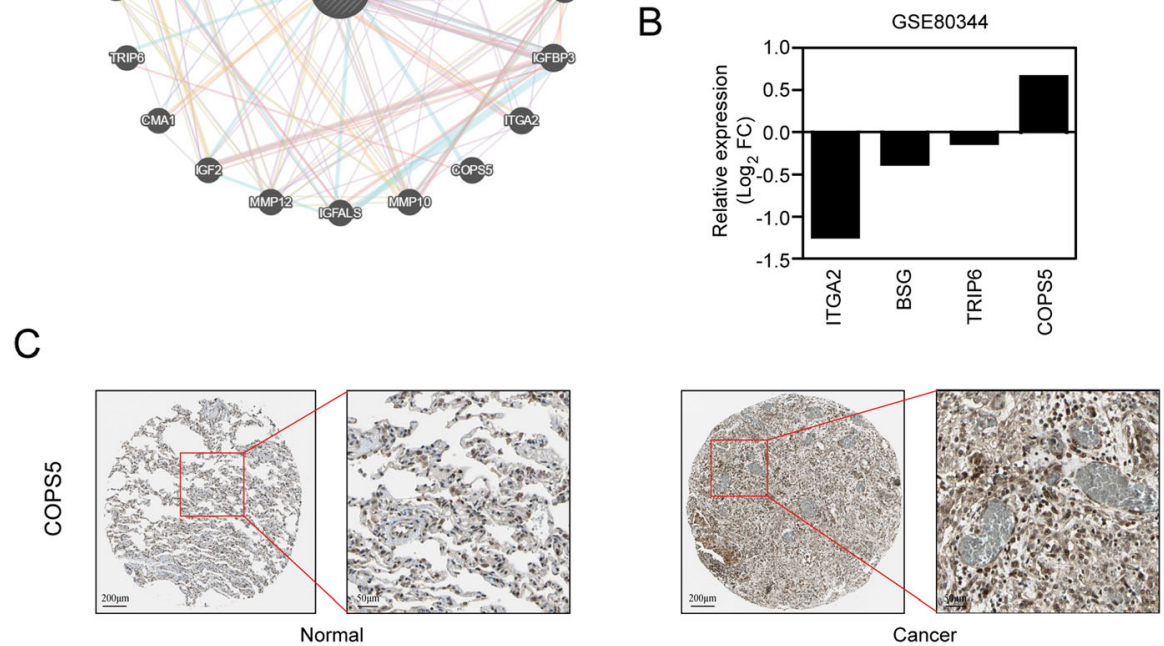

D
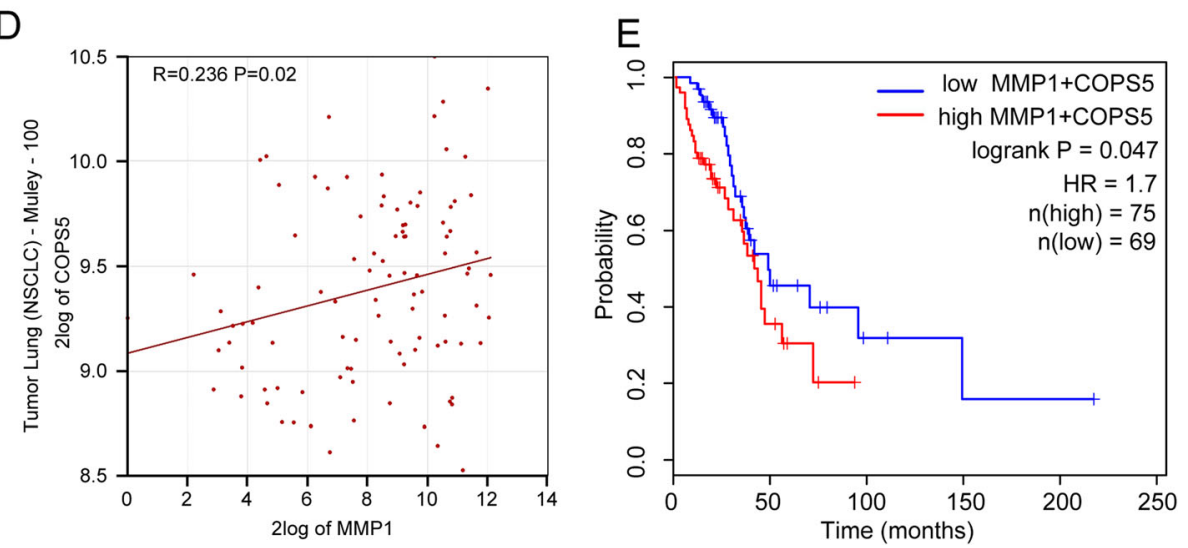

Fig. 4 Validation of MMP1 hub genes related to erlotinib resistance in NSCLC. a Proteins/genes interaction network of MMP1 created by GeneMANIA. The network legends refer to the interaction types (illustrated in the networks) between proteins/genes. $\mathbf{b}$ Relative mRNA level of 4 genes interacting with MMP1 based on the microarray data of GSE80344. $\mathbf{c}$ Representative immunohistochemistry staining of COPS5 expression in normal lung tissues and NSCLC tissues. d Positive correlation between MMP1 and COPS5 expression in the Tumor Lung $(R=0.236, P=0.02)$. e The prognostic value was obtained from the GEPIA2 database when both MMP1 and COPS5 were highly expressed $(H R=1.7, P=0.047)$

immunohistochemical results indicated that the expression level of COPS5 exhibited significant upregulation in NSCLC tissues (Fig. 4c). Meanwhile analysis of the Tumor Lung (NSCLC)-Muley-100 dataset by R2 online tool revealed that MMP1 expression was positively correlated with COPS5 (Fig. $4 \mathrm{~d} ; R=0.236, P=0.02$ ). And we further performed the survival analysis by GEPIA2 and found that patients with simultaneous high expression of MMP1 and COPS5 were associated with poor overall survival $(\mathrm{HR}=1.7, \log$-rank $P=0.047)$ (Fig. 4e). Thus, COPS5 might be involved in MMP1-mediated erlotinib resistance.

\section{Validation of the Erlotinib resistance function of MMP1}

To further investigate the mechanism of erlotinib resistance in NSCLC, the transcription factors targeting MMP1 were explored by FunRich analysis, HOXA9, PBX1, JUND, JUNB, and JUN were identified to be the top five transcription factors which targeting MMP1 (Fig. 5a). Among these transcription factors, HOXA9 $[13,36]$ and PBX1 $[37,38]$ have been reported to function as drivers in drug resistance of multiple cancer types. Finally, the biological process of MMP1 was annotated using the Coremine Medical Database. As shown in Fig. 5b, 10 biological processes were closely associated 


\section{A}

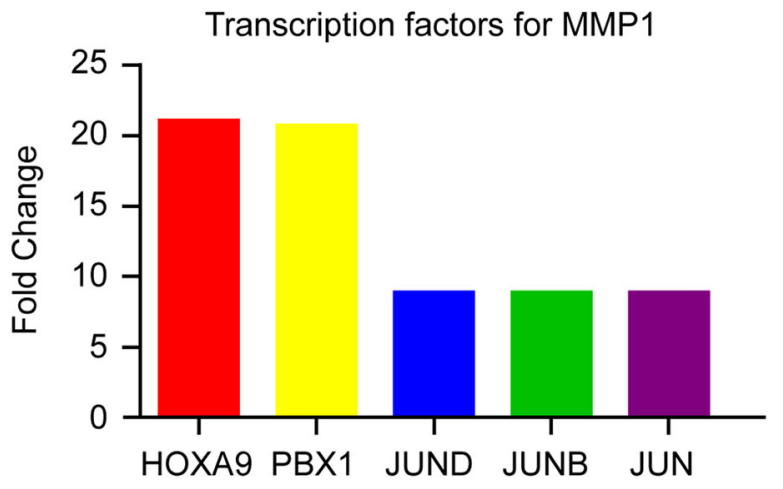

B

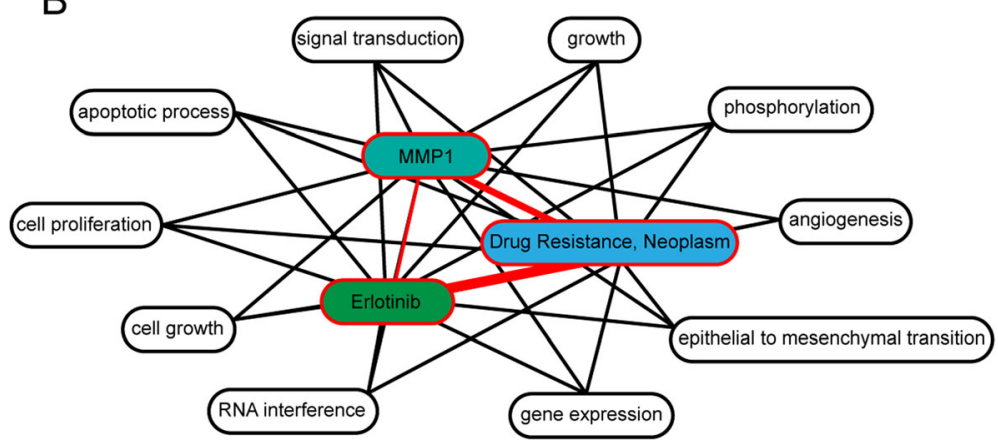

Fig. 5 Validation of the erlotinib resistance function of MMP1. a Relative expression of transcription factors target MMP1 including HOXA9, PBX1, $J U N D$, JUNB, and JUN. b Annotation of biological processes of MMP1 with erlotinib and drug resistance using the Coremine Medical online tool

with drug resistance, which MMP1 may affect drug resistance by regulating cell growth, apoptosis, protein phosphorylation, and angiogenesis. Consequently, these results indicated that MMP1 may confer the erlotinib resistance in NSCLC through a variety of mechanisms.

\section{Discussion}

Conventional chemotherapy and molecular-targeted therapy are currently two important strategies for NSCL $\mathrm{C}$ treatment [9]. Whereas, the clinical prognosis of both strategies is poor due to the subsequently acquired drug resistance, whose mechanisms have been partly reported, such as the drug target genetic mutations $[39,40]$. The significance of EGFR has been identified in NSCLC, and consequently promotes the application of EGFR inhibitors [41]. Erlotinib is the first generation of EGFR inhibitors, and it has been commonly used in locally advanced or metastatic NSCLC after chemotherapy failure [10]. Although the efficacy of erlotinib in NSCLC has been confirmed, long course application of erlotinib inevitably leads to drug resistance and tumor relapse [42, 43]. At present, gene identifications conferring drug resistance through high-throughput screening methods and the design of drugs aiming at these genes may be a promising appeal in cancer treatment $[8,44]$. For example, LEE011, a CDK 4/6 inhibitor, has a good inhibitory effect on the resistance caused by PI3K mutation in breast cancer [45]. In the present study, we combined an NSCLC erlotinib resistant microarray (GSE80344) with an NSCLC tissue Dataset (GSE19188), and initially identified MMP1 as a candidate gene conferring erlotinib resistance in NSCLC based on the comprehensive bioinformatics analyses.

Matrix metalloproteinases (MMPs) family is a zincdependent peptidase, which is responsible for the degradation of the extracellular matrix (ECM) [46]. Moreover, MMPs play a key role in tissue remodeling and regulation in various diseases containing arthritis, cirrhosis, and cancers [47, 48]. Among them, MMP1 has been proved to be highly expressed in various kinds of cancers, mainly acting to degrade type I and type III collagen in the extracellular environment [49]. It has been reported that MMP1 enhanced tumor cell invasiveness by increasing vascular endothelial growth factor (VEGF) and bone morphogenetic protein 2/4 [50-52]. In addition, Slug enhanced MMP1 transcription by directly binding to the promoter region of breast cancer cells, resulting in multiple drug resistance [53]. Additionally, it has been reported that MMP1 can be applied as a candidate therapeutic target and biomarker for drug response 
$[17,54,55]$. In the present study, we revealed that MMP1 was overexpressed in NSCLC tissues and erlotinib-resistant NSCLC cells, and it negatively associated with overall survival in NSCLC patients. Through using protein/gene interactions (GeneMANIA), biological process annotation (Coremine) and transcription factor analysis (FunRich), we further verified that MMP1 may confer the erlotinib resistance in NSCLC through various mechanisms. GeneMANIA results indicated that MMP1 was closely associated with 20 genes, including BSG, TRIP6, ITGA2, F2R, MMP3, COPS5, etc. And four genes (ITGA2, BSG, TRIP6, and COPS5) have been found to regulate drug resistance in different cancer models [56-59]. Especially, COPS5 was significantly upregulated in the erlotinib resistance dataset GSE80344 and NSCLC tissues. And the survival analysis indicated that patients with simultaneous high expression of MMP1 and COPS5 were associated with poor overall survival. It has been reported that COPS5 plays a crucial role in tamoxifen resistance in oestrogen receptor $\alpha$ $(E R \alpha)$ positive breast cancer patients, through regulating NCoR ubiquitination-proteasomal degradation. Other studies revealed that the expression of MMP1 was positively correlated with COPS5 [60]. These results demonstrated that COPS5 might be involved in MMP1mediated erlotinib resistance. Several transcription factors in the epigenetic regulation of MMP1 were also screened in the present study, and two major transcription factors, HOXA9 and PBX1, were identified. Previous studies have also reported that HOXA9 and PBX1 were involved in drug resistance regulation [36, 37], suggesting that HOXA9 and PBX1 may play an important role in erlotinib resistance through regulating the transcription of MMP1. Taken together, we validated that MMP1 is a potential erlotinib resistance gene in NSCLC and could be a prognostic biomarker for erlotinib treatment.

\section{Conclusion}

In conclusion, we demonstrated that MMP1 may confer the erlotinib resistance in NSCLC through multiple mechanisms based on comprehensive bioinformatics analyses. Our study provides a potential target for reversion and prognosis of erlotinib resistance in NSCLC, and further studies will clarify the underlying molecular mechanism by which MMP1 regulates erlotinib resistance.

\section{Supplementary information}

Supplementary information accompanies this paper at https://doi.org/10. 1186/s41065-020-00145-x.

Additional file 1: Supplementary Table 1. Identification of DEGs. Additional file 2: Supplementary Table 2. GO enrichment analysis.
Additional file 3: Supplementary Table 3. KEGG pathway analysis. Additional file 4: Supplementary Table 4. Identification of DEGs. Additional file 5: Supplementary Table 5. GO enrichment analysis. Additional file 6: Supplementary Table 6. KEGG pathway analysis.

\section{Abbreviations}

BP: Biological process; BSG: Basigin; CC: Cellular component; COPS5: COP9 signalosome subunit 5; DAVID: Visualization and Integrated Discovery; DEGs: Differentially expressed genes; ECM: Extracellular matrix; EGFR: Epidermal growth factor receptor; ERa: Oestrogen receptor a; FC: Fold change; GEO: Gene Expression Omnibus; GO: Gene Ontology; HPA: The Human Protein Atlas; ITGA2: Integrin subunit alpha 2; KEGG: Kyoto Encyclopedia of Genes and Genomes; KM: Kaplan-Meier; LUAD: Lung adenocarcinoma; LUSC: Lung squamous cell carcinoma; MAPK: Mitogenactivated protein kinase; MF: Molecular function; MMP1: Matrix metalloproteinase 1; NSCLC: Non-small cell lung cancer; STAT3: Signal transducer and activator of transcription 3; TRIP6: Thyroid hormone receptor interactor 6; VEGF: Vascular endothelial growth factor

\section{Acknowledgements}

Not applicable.

Authors' contributions

HYZ and QMX analyzed the data and wrote the manuscript. CPH and JZ collected the data. QZ and RZ reviewed the manuscript. All authors read and approved the manuscript.

\section{Funding}

This study was supported by a grant from the National Natural Science Foundation of China (Grant No. 81874357).

Availability of data and materials

The datasets analyzed during the current study are available in the GEO database (https://www.ncbi.nlm.nih.gov/geo).

Consent for publication

Not applicable.

\section{Competing interests}

The authors declare that they have no competing interests.

\section{Author details}

${ }^{1}$ Department of Pharmacy, the Second Affiliated Hospital of Army Medical University, 83 Xinqiao Road, Chongqing 400037, China. ${ }^{2}$ Maternity service center of Beijing Fengtai District Maternal and Child health care hospital, Beijing 100067, China.

Received: 28 March 2020 Accepted: 16 July 2020

Published online: 23 July 2020

\section{References}

1. Chen W, et al. Cancer statistics in China, 2015. CA Cancer J Clin. 2016;66(2): 115-32.

2. Siegel RL, Miller KD, Jemal A. Cancer statistics, 2018. CA Cancer J Clin. 2018; 68(1):7-30.

3. Ramalingam SS, Owonikoko TK, Khuri FR. Lung cancer: new biological insights and recent therapeutic advances. CA Cancer J Clin. 2011;61(2):91112.

4. Rami-Porta R, et al. Lung cancer - major changes in the American joint committee on Cancer eighth edition cancer staging manual. CA Cancer J Clin. 2017:67(2):138-55.

5. Meza $R$, et al. Lung cancer incidence trends by gender, race and histology in the United States, 1973-2010. PLoS One. 2015;10(3):e0121323.

6. Arriagada $\mathrm{R}$, et al. Adjuvant chemotherapy, with or without postoperative radiotherapy, in operable non-small-cell lung cancer: two meta-analyses of individual patient data. Lancet. 2010;375(9722):1267-77.

7. Cersosimo RJ. Lung cancer: a review. Am J Health Syst Pharm. 2002;59(7): 611-42. 
8. Gao H, et al. High-throughput screening using patient-derived tumor xenografts to predict clinical trial drug response. Nat Med. 2015;21(11) 1318-25.

9. Hirsch FR, et al. New and emerging targeted treatments in advanced nonsmall-cell lung cancer. Lancet. 2016;388(10048):1012-24.

10. Minna, JD and J. Dowell. Erlotinib hydrochloride. Nat Rev Drug Discov. 2005: Suppl: S14-5.

11. Shepherd FA, et al. Erlotinib in previously treated non-small-cell lung cancer. N Engl J Med. 2005;353(2):123-32

12. Yang $Z$, et al. Comparison of gefitinib, erlotinib and afatinib in non-small cell lung cancer: a meta-analysis. Int J Cancer. 2017;140(12):2805-19.

13. Pojo $M$, et al. A transcriptomic signature mediated by HOXA9 promotes human glioblastoma initiation, aggressiveness and resistance to temozolomide. Oncotarget. 2015;6(10):7657-74.

14. Cardona AF, et al. Acquired resistance to Erlotinib in EGFR mutation-positive lung adenocarcinoma among Hispanics (CLICaP). Target Oncol. 2017;12(4): 513-23.

15. Kosaka T, et al. Analysis of epidermal growth factor receptor gene mutation in patients with non-small cell lung cancer and acquired resistance to gefitinib. Clin Cancer Res. 2006;12(19):5764-9.

16. Cevenini A, Orru S. Molecular Signatures of the Insulin-like Growth Factor 1mediated Epithelial-Mesenchymal Transition in Breast, Lung and Gastric Cancers. Int J Mol Sci. 2018;19(8):2411.

17. Jakobsen KR, et al. The role of epithelial to mesenchymal transition in resistance to epidermal growth factor receptor tyrosine kinase inhibitors in non-small cell lung cancer. Transl Lung Cancer Res. 2016;5(2):172-82.

18. Lee $Y$, et al. Inhibition of IGF1R signaling abrogates resistance to afatinib (BIBW2992) in EGFR T790M mutant lung cancer cells. Mol Carcinog. 2016; 55(5):991-1001.

19. Nurwidya F, et al. Treatment with insulin-like growth factor 1 receptor inhibitor reverses hypoxia-induced epithelial-mesenchymal transition in non-small cell lung cancer. Biochem Biophys Res Commun. 2014;455(3-4): 332-8.

20. Witta SE, et al. Restoring E-cadherin expression increases sensitivity to epidermal growth factor receptor inhibitors in lung cancer cell lines. Cancer Res. 2006:66(2):944-50.

21. Kobayashi $\mathrm{S}$, et al. EGFR mutation and resistance of non-small-cell lung cancer to gefitinib. N Engl J Med. 2005;352(8):786-92.

22. Goldstein DM, Gray NS, Zarrinkar PP. High-throughput kinase profiling as a platform for drug discovery. Nat Rev Drug Discov. 2008;7(5):391-7.

23. Kulasingam V, Pavlou MP, Diamandis EP. Integrating high-throughput technologies in the quest for effective biomarkers for ovarian cancer. Nat Rev Cancer. 2010;10(5):371-8.

24. Chen F, et al. Gene expression profile for predicting survival of patients with meningioma. Int J Oncol. 2015;46(2):791-7.

25. Kulasingam V, Diamandis EP. Strategies for discovering novel cancer biomarkers through utilization of emerging technologies. Nat Clin Pract Oncol. 2008;5(10):588-99.

26. Lu Y, et al. A gene expression signature predicts survival of patients with stage I non-small cell lung cancer. PLoS Med. 2006;3(12):e467.

27. Dutkowski J, et al. A gene ontology inferred from molecular networks. Nat Biotechnol. 2013;31(1):38-45.

28. Kanehisa M, et al. KEGG: new perspectives on genomes, pathways, diseases and drugs. Nucleic Acids Res. 2017;45(D1):D353-d361.

29. Lanczky A, et al. miRpower: a web-tool to validate survival-associated miRNAs utilizing expression data from 2178 breast cancer patients. Breast Cancer Res Treat. 2016;160(3):439-46.

30. Franz M, et al. GeneMANIA update 2018. Nucleic Acids Res. 2018;46(W1): W60-w64.

31. Pathan $\mathrm{M}$, et al. FunRich: an open access standalone functional enrichment and interaction network analysis tool. Proteomics. 2015;15(15):2597-601.

32. Lee DH. Treatments for EGFR-mutant non-small cell lung cancer (NSCLC): the road to a success, paved with failures. Pharmacol Ther. 2017;174:1-21.

33. Gammelgaard KR, et al. Up-regulated FGFR1 expression as a mediator of intrinsic TKI resistance in EGFR-mutated NSCLC. Transl Oncol. 2019;12(3):432-40.

34. Masuda C, et al. Bevacizumab counteracts VEGF-dependent resistance to erlotinib in an EGFR-mutated NSCLC xenograft model. Int J Oncol. 2017; 51(2):425-34.

35. Shintani $T$, et al. Eukaryotic translation initiation factor 3 subunit $C$ is associated with acquired resistance to erlotinib in non-small cell lung cancer. Oncotarget. 2018;9(101):37520-33.
36. Ju T, et al. Overexpression of NAC1 confers drug resistance via HOXA9 in colorectal carcinoma cells. Mol Med Rep. 2017:16(3):3194-200.

37. Abe M, et al. Internal tandem duplication in FLT3 attenuates proliferation and regulates resistance to the FLT3 inhibitor AC220 by modulating p21Cdkn1a and Pbx1 in hematopoietic cells. PLoS One. 2016;11(7): e0158290.

38. Jung JG, et al. Ovarian Cancer Chemoresistance relies on the stem cell reprogramming factor PBX1. Cancer Res. 2016;76(21):6351-61.

39. Longley DB, Johnston PG. Molecular mechanisms of drug resistance. $J$ Pathol. 2005;205(2):275-92.

40. Misale $\mathrm{S}$, et al. Emergence of KRAS mutations and acquired resistance to anti-EGFR therapy in colorectal cancer. Nature. 2012;486(7404):532-6.

41. Paez JG, et al. EGFR mutations in lung cancer: correlation with clinical response to gefitinib therapy. Science. 2004;304(5676):1497-500.

42. Horiike $A$, et al. Phase II study of erlotinib for acquired resistance to gefitinib in patients with advanced non-small cell lung cancer. Anticancer Res. 2014; 34(4):1975-81.

43. Zhu CQ, et al. Role of KRAS and EGFR as biomarkers of response to erlotinib in National Cancer Institute of Canada clinical trials group study BR.21. J Clin Oncol. 2008:26(26):4268-75.

44. Yeung KT, et al. Circulating tumor DNA for mutation detection and identification of mechanisms of resistance in non-small cell lung Cancer. Mol Diagn Ther. 2017;21(4):375-84.

45. Vora SR, et al. CDK 4/6 inhibitors sensitize PIK3CA mutant breast cancer to PI3K inhibitors. Cancer Cell. 2014;26(1):136-49.

46. Kapoor $\mathrm{C}$, et al. Seesaw of matrix metalloproteinases (MMPs). J Cancer Res Ther. 2016;12(1):28-35.

47. Conlon GA, Murray $\mathrm{Gl}$. Recent advances in understanding the roles of matrix metalloproteinases in tumour invasion and metastasis. J Pathol. 2019; 247(5):629-40.

48. Herzog C, Haun RS, Kaushal GP. Role of meprin metalloproteinases in cytokine processing and inflammation. Cytokine. 2019;114:18-25.

49. Diniz-Fernandes T, et al. Matrix metalloproteinase-1 (MMP-1) and (MMP-8) gene polymorphisms promote increase and remodeling of the collagen III and $V$ in posterior tibial tendinopathy. Histol Histopathol. 2018;33(9):929-36.

50. Mazor R, et al. Matrix metalloproteinase-1-mediated up-regulation of vascular endothelial growth factor-2 in endothelial cells. J Biol Chem. 2013; 288(1):598-607.

51. Su YP, et al. Leptin induces MMP1/13 and ADAMTS 4 expressions through bone morphogenetic protein-2 autocrine effect in human chondrocytes. Cell Biochem. 2018;119(4):3716-24.

52. Tratwal J, et al. Influence of vascular endothelial growth factor stimulation and serum deprivation on gene activation patterns of human adipose tissue-derived stromal cells. Stem Cell Res Ther. 2015:6:62.

53. Shen CJ, et al. MMP1 expression is activated by slug and enhances multidrug resistance (MDR) in breast cancer. PLoS One. 2017:12(3):e0174487.

54. Kim E, et al. Capicua suppresses hepatocellular carcinoma progression by controlling the ETV4-MMP1 axis. Hepatology. 2018;67(6):2287-301.

55. Pei D. Matrix metalloproteinases target protease-activated receptors on the tumor cell surface. Cancer Cell. 2005:7(3):207-8.

56. Lu R, et al. COPS5 amplification and overexpression confers tamoxifenresistance in ERalpha-positive breast cancer by degradation of NCoR. Nat Commun. 2016;7:12044

57. Miao X, et al. Overexpression of TRIP6 promotes tumor proliferation and reverses cell adhesion-mediated drug resistance (CAM-DR) via regulating nuclear p27(Kip1) expression in non-Hodgkin's lymphoma. Tumour Biol. 2016:37(1):1369-78.

58. Wu X. Up-regulation of YPEL1 and YPEL5 and down-regulation of ITGA2 in erlotinib-treated EGFR-mutant non-small cell lung cancer: a bioinformatic analysis. Gene. 2018;643:74-82.

59. Zou W, et al. Hepatitis B X-interacting protein promotes cisplatin resistance and regulates CD147 via Sp1 in ovarian cancer. Exp Biol Med (Maywood). 2017;242(5):497-504

60. Levinson $\mathrm{H}$, et al. Alpha $\mathrm{V}$ integrin prolongs collagenase production through Jun activation binding protein 1. Ann Plast Surg. 2004;53(2):155-61.

\section{Publisher's Note}

Springer Nature remains neutral with regard to jurisdictional claims in published maps and institutional affiliations. 